\title{
Welcome to the year 2019
}

Nadia Magnenat-Thalmann ${ }^{1}$

(c) Springer-Verlag GmbH Germany, part of Springer Nature 2018

The Visual Computer Journal is going well although unfortunately, its impact factor has decreased from 1.468 to 1.036 . It is difficult to know why the citations have lowered, but one of the reasons might be that we have received more regular submissions during the year 2018. The Visual Computer has received more than 800 submissions in $2018,11.5 \%$ more than in 2017. This is a tremendous achievement from authors, associate editors and reviewers, and it may be more difficult to keep the impact factor with so many papers.

Each submitted paper is handled by an associate editor (AE) who finds at least 3 reviewers to evaluate a paper. I take the opportunity to warmly thank the associate editors as well as all reviewers listed at the end of the issue for their tremendous and invaluable work.

Throughout 2018, some associate editors have left the editorial board of the Visual Computer. I would like to thank them for their great work and contribution.

Here is the list of the associate editors who have left the editorial board:

- Shahar Kovalsky, Duke University, USA.

- Jingyi Yu, University of Delaware, USA.

- Richard Hao Zhang, Simon Fraser University, Canada.

- Guillaume Lavoue, LIRIS, CNRS, France.

- Yaron Lipman, Weizmann Institute of Science, Israel.

- Hong Qin, State University of New York at Stony Brook, USA.

- Prosenjit Bose, Carleton University, Canada.

- Enhua Wu, University of Macau and Academia Sinica, Macau, China.

- Junfeng Yao, Xiamen University, China.

- Yasuyuki Matsushita, Osaka University, Japan.

- Zerrin Yumak, University of Utrecht, Netherlands.

- Daniel Weiskopf, University of Stuttgart, Germany.

- Frederic Fol Leymarie, Goldsmiths, United Kingdom.

Nadia Magnenat-Thalmann

thalmann@miralab.ch

1 MIRALab-CUI, University of Geneva, Battelle, Building A, 7, Route de Drize, 1227 Carouge, Geneva, Switzerland
The editorial board of the Visual Computer is renewed regularly, and we welcome our new associate editors listed below:

- Benjamin Bustos, University of Chile, Chile.

- Daniel Keim, University of Konstanz, Germany.

- Juyong Zhang, University of Science and Technology of China, China.

- Ruizhen Hu, Shenzhen University, China.

- Renjie Chen, Max-Planck Institute for Informatics, Germany.

- Filip Sadlo, Heidelberg University, Germany.

- Ofir Weber, Bar-Ilan University, Israel.

- Junhui Hou, City University of Hong Kong, Hong Kong.

- Xifeng Gao, Florida State University, USA.

- Andreas Aristidou, University of Cyprus, Cyprus.

- Anne Verroust-Blondet, INRIA, France.

During 2018, the journal published 37 best papers of the Computer Graphics Conference (CGI'2018) that was held in Bintan, Indonesia, in June 2018. During the year 2018, best papers from other conferences such as EuroVA 2017 and Cyberworlds 2017 were extended, and some of them, after review, were accepted for publication in the Visual Computer.

I would like to thank the Editorial Assistants Dr Nisha Jain and Dr Manoj Ramanathan for their collaboration in the management of the journal.

Finally, this journal could not exist without the strong support of our Editorial Senior Director at Springer, Beverley Ford, and our Editorial Director Rachel Moriarty. We would like to address our special thanks to Beverly Ford who has retired this year. We appreciated her strong support all over these years. She used to be a tremendous Senior Editor, and we will miss her a lot. 
We would also like to thank Beate Uhl, the Production Editor, and Vidhya Velayudhan, from the journal editorial office, for their continuous support and great assistance.Lastly, a big thank goes to all authors who have submitted their work to the Visual Computer as well as to the readers of the Visual Computer. Without them, the journal could not exist.
We hope that 2019 will be a prosperous year for the Visual Computer, and we encourage authors to submit their best work.

Publisher's Note Springer Nature remains neutral with regard to jurisdictional claims in published maps and institutional affiliations. 